Original Article

\title{
Improved underpants to reduce toileting problems in patients with poor standing balance
}

\author{
Yuji Koike $^{1)^{*}}$, Shuhei KoedA ${ }^{1)}$, Koshi Sumigawa ${ }^{1)}$, Hitoshi Tsushima ${ }^{1)}$ \\ 1) Graduate School of Health Sciences, Hirosaki University: 66-1 Honcho, Hirosaki, Aomori 036-8564, \\ Japan
}

\begin{abstract}
Purpose] We developed and assessed improved underpants allowing patients to easily and completely open the crotch by pulling upward a string on the back of the underpants while seated. [Subjects and Methods] Healthy subjects tested commercial open-crotch underpants for disabled people and our improved open-crotch underpants by opening the crotch while seated. Measurement parameters included the percentage of crotch opening and the degree of genital or anal exposure before and after opening the crotch, as well as fit. The percentage of crotch opening and the degree of genital or anal exposure were recorded with a digital camera, and fit was determined with a visual analog scale. The measurements for both types of underpants were then compared. [Results] The commercial underpants for disabled people allowed opening of the crotch while seated, but the exposure of the genital and anal areas was insufficient to allow waste elimination. The improved underpants allowed sufficient opening of the crotch while seated and adequately exposed the genital and anal areas. The fit score of the improved underpants was significantly better than that of the commercial underpants. [Conclusion] The improved opencrotch underpants may allow for more effective opening of the crotch while seated than commercial open-crotch underpants for disabled people.

Key words: Stroke, Toileting, Seated position
\end{abstract}

(This article was submitted Sep. 9, 2014, and was accepted Oct. 10, 2014)

\section{INTRODUCTION}

Stroke patients often have toileting problems, and once such problems occur and the disabled individual requires toileting care, he or she may be burdened psychologically ${ }^{1)}$. If they need help with toileting, it is hard for them to live at home ${ }^{2,3)}$. Therefore, establishing toileting independence is a critically important issue for stroke patients, and many occupational therapists have dealt with this issue ${ }^{4)}$.

The toileting activities that occupational therapists work on comprise various movements, namely, "maintaining perineal hygiene" and "adjusting clothing after toileting"5). "Adjusting clothing after toileting", referred to as "pulling the lower garments up and down", is reported to comprise the most difficult movements ${ }^{6,7)}$. Pulling the lower garments up and down is generally performed in a standing position, and patients who have balance and stability issues while standing often face difficulty with this task. Occupational therapists are expected to suggest ways to help such stroke patients pull their lower garments up and down independently. Currently available methods for patients with poor standing balance include pulling the lower garments up

*Corresponding author. Yuji Koike (E-mail: ot_koike@ hirosaki-u.ac.jp)

C2015 The Society of Physical Therapy Science. Published by IPEC Inc. This is an open-access article distributed under the terms of the Creative Commons Attribution Non-Commercial No Derivatives (by-ncnd) License $<$ http://creativecommons.org/licenses/by-nc-nd/3.0/>. and down while seated ${ }^{8,9)}$ and the use of open-crotch underpants that allow waste elimination while seated. Pulling the lower garments up and down while seated requires high functionality and capability, and few stroke patients can successfully perform the technique ${ }^{9}$. Commercial open-crotch underpants (TacaoF Co., Ltd., Osaka, Japan) ${ }^{10)}$ for disabled people are not often used in clinical settings, and there have been very few reports on their use. Possible reasons for this lack of use are that although commercial open-crotch underpants for disabled people are designed to allow the crotch to be opened while seated, the opening is actually insufficient to allow waste elimination. Moreover, the pants are often uncomfortable because of poor fit. New types of underpants designed to overcome these problems may allow patients with poor standing balance to pull their lower garments up and down independently. Therefore, we improved the design used in one type of commercial open-crotch underpants (Fig. 1) to address the problems of insufficient opening of the crotch, insufficient genital and anal exposure, and poor fit. Our improvement was to add a mechanism to open the crotch by pulling two handles attached to strings placed on either side of the back of the underpants. We also experimentally produced better-fitting underpants (hereinafter referred to as "improved open-crotch underpants") by referring to the structure and size of underpants for healthy individuals (Fig. 2).

The improved open-crotch underpants were designed to expose the genital and anal areas, allowing patients to eliminate waste without soiling their underpants, through a two-step action as follows: 1) open one side of the crotch by 


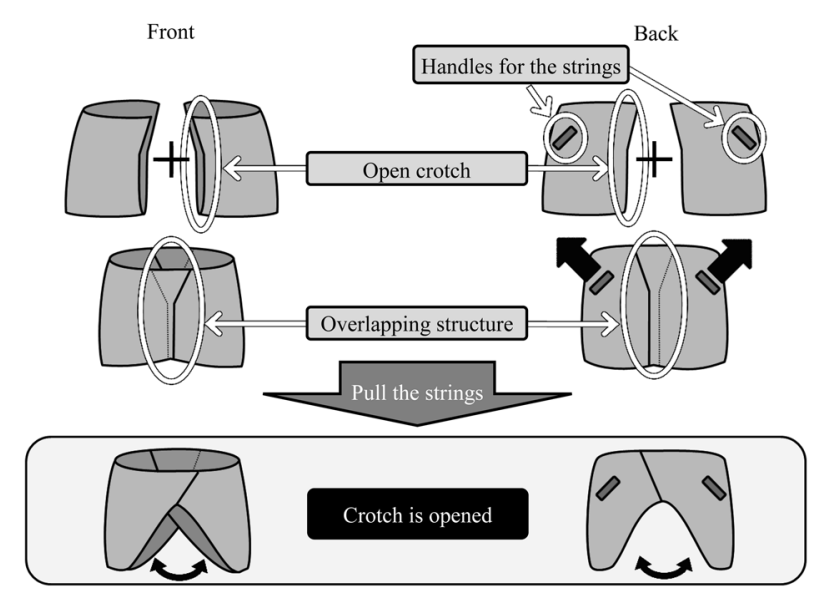

Fig. 1 Structure of the improved open-crotch underpants

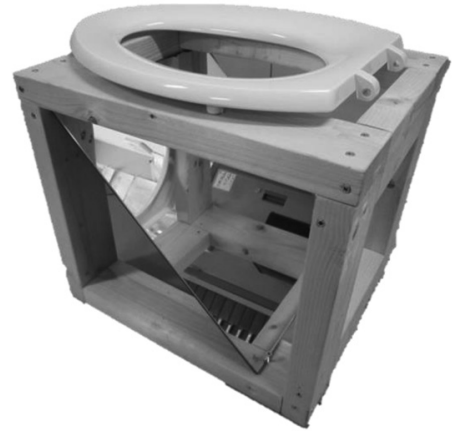

Fig. 3. Chair with toilet seat and mirror used to measure the degree of crotch opening and genital and anal exposure

pulling upward on one string at the back of the underpants with the buttock on that side lifted up slightly from the toilet seat, and 2) open the other side of the crotch by pulling upward on the other string at the back of the underpants, with the buttock on that side lifted up slightly from the toilet seat. We evaluated the effectiveness of the underpants by examining the degree of opening of the crotch and the degree of genital and anal exposure in healthy subjects.

\section{SUBJECTS AND METHODS}

Five healthy males (age range, 22 to 32 years $(24.0 \pm$ 4.5); mean height, $170.9 \pm 3.5 \mathrm{~cm}$ ) who provided written informed consent to participate were included in the present study. The study was approved by the Committee of Medical Ethics of the Hirosaki University Graduate School of Medicine, Hirosaki, Japan (reference number 2012-276).

To start, the subject sat on a chair fitted with a toilet seat and mirror, with the trunk vertical and the feet planted firmly on the floor. The task to open the crotch from this position was performed by either opening the thighs (commercial open-crotch underpants) or by pulling the strings at the back of the underpants (improved open-crotch underpants). All subjects performed each movement three times after practic-



Fig. 2. Structures of the commercial open-crotch underpants and our improved open-crotch underpants

ing several times. A chair with a toilet seat and mirror (Fig. 3) was created to measure the degree of crotch opening and genital and anal exposure. The anal area could be observed from behind as a reflection in the mirror. The degree of crotch opening and genital or anal exposure were recorded with a digital camera from behind the chair before and after the crotch-opening task. In addition, the subjects' subjective impression of the fit of the underpants was measured with a visual analog scale (VAS). All subjects were asked to wear each type of underpants and to judge the fit by placing a check mark on a $100-\mathrm{mm}$ VAS, where $0.0 \mathrm{~mm}$ represented the "worst fit" and $100.0 \mathrm{~mm}$ represented the "best fit".

Using image-processing software (Adobe Photoshop CS6 Extended, Adobe Japan, Tokyo, Japan), photos obtained with a digital camera were processed to determine the area of crotch opening and the inner area of the toilet seat. These measurements were then used to calculate the percentage crotch opening by dividing the former area by the latter. The mean values of three measurements of the percentage crotch opening before and after the crotch-opening task were used for analysis. The percentage of crotch opening for each type of underpants was compared before and after the crotchopening task. We also compared the percentage of crotch opening between the two types of underpants before and after the crotch-opening task. Simultaneously, we assessed the degree of genital and anal exposure.

To compare the fit of the commercial and improved open-crotch underpants, we used the VAS data to measure the distance from "worst fit" to the check mark on the VAS scale. The distance value was expressed as a percentage to calculate the fit of the underpants for comparison with a higher fit value indicating that the underpants fit better.

For statistical analysis, we used Wilcoxon's signed rank test to compare the percentage of crotch opening before and after the crotch-opening task in both types of underpants; we also used it to compare the fit between the two types of underpants. SPSS Statistics 17.0 (SPSS Japan Inc., Tokyo, Japan) was used as the software for statistical analysis, and 
Table 1. Comparison of the percentage of crotch opening between before and after the crotch-opening task in the two types of underpants

\begin{tabular}{lcc}
\hline & $\begin{array}{c}\text { Commercial open-crotch } \\
\text { underpants }\end{array}$ & $\begin{array}{c}\text { Improved open-crotch } \\
\text { underpants }\end{array}$ \\
\hline $\begin{array}{l}\text { Before the crotch-opening task } \\
\text { After the crotch-opening task }\end{array}$ & $2.40(0.89-8.04)$ & $0.00(0.00-0.00)^{\dagger}$ \\
\hline
\end{tabular}

Numbers are medians (25-75\%), and comparisons were made using Wilcoxon's signed rank test. Comparison of the percentage of crotch opening before and after the crotchopening task: $* \mathrm{p}<0.05$.

Comparison of the percentage of crotch opening between the two types of underpants: $\dagger \mathrm{p}<0.05$.



Improved
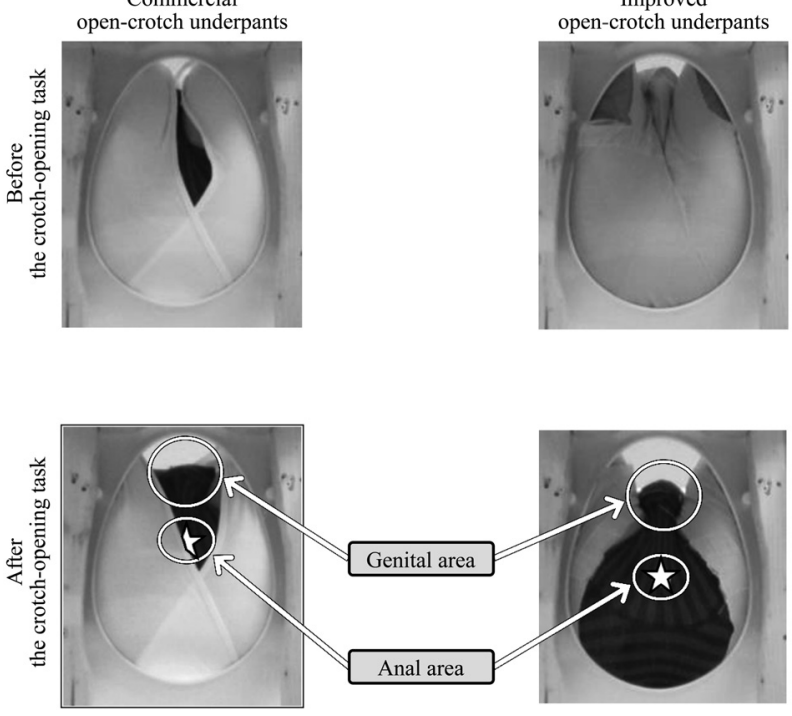

Fig. 4. Comparison of genital and anal exposure in both types of underpants

the significance level was set at $\mathrm{p}<0.05$.

\section{RESULTS}

The results for the percentage of crotch opening before and after the crotch-opening task are shown in Table 1. The percentage of crotch opening for both the commercial and improved open-crotch underpants was significantly larger after crotch opening than before $(p<0.05)$. Next, we compared the percentage of crotch opening between the two types of underpants. The percentage of crotch opening before the crotch-opening task was significantly larger in the commercial open-crotch underpants than in the improved open-crotch underpants $(\mathrm{p}<0.05)$. In contrast, the percentage after crotch opening was significantly larger in the improved open-crotch underpants than in the commercial open-crotch underpants $(\mathrm{p}<0.05)$.

Next, we compared the degree of genital and anal exposure (Fig. 4). Before the crotch-opening task, neither the genital nor the anal region was exposed in either type of underpants. After the crotch-opening task, the genital exposure was sufficiently large to allow waste elimination in the commercial open-crotch underpants, but the anal exposure was
Table 2. Comparison of fit between the two types of underpants

\begin{tabular}{ccc}
\hline & $\begin{array}{c}\text { Commercial open-crotch } \\
\text { underpants }\end{array}$ & $\begin{array}{c}\text { Improved open-crotch } \\
\text { underpants }\end{array}$ \\
\hline Fit score & $39.0(37.0-57.0)$ & $70.0(66.0-74.0)^{*}$ \\
\hline
\end{tabular}

Numbers are medians (25-75\%), and comparisons were made using Wilcoxon's signed rank test.

Comparison of fit: $* p<0.05$.

insufficient. In contrast, both genital and anal exposure were sufficiently large to allow waste elimination in the improved open-crotch underpants.

We also compared the fit of the two types of underpants (Table 2). The VAS scores for fit of the improved opencrotch underpants was significantly better than that of the commercial open-crotch pants $(\mathrm{p}<0.05)$.

\section{DISCUSSION}

The results of the present study showed that the commercial open-crotch underpants for disabled people ${ }^{10)}$ allowed the crotch to open while seated, but the exposure of the anal area was insufficient to allow waste elimination. This means that toileting while wearing the commercial open-crotch underpants is likely to be problematic and unsanitary. In addition, the crotch was opened simply by the act of sitting and was therefore always open. This means that wearing these underpants revealed the wearer's skin, potentially giving them feelings of shame. Possible reasons for the crotch opening insufficiently exposing the anal area and for the constant opening of the crotch while seated were poor fit and the large crotch overlap.

The crotch opening in the improved underpants was closed before the crotch-opening task but it opened to sufficiently expose the genital and anal areas after the crotchopening task. This means that if patients wear the improved underpants during toileting, it is possible to avoid soiling the underpants. Possible reasons for this sufficient crotch opening were minimization of the overlap area, proper fit, and the mechanism for crotch opening that requires pulling on strings attached to the back of the underpants. The improved underpants allowed sufficient opening of the crotch while seated and may therefore be more effective than the commercial open-crotch underpants.

There are two problems with the improved open-crotch underpants. The first problem was that stroke patients may 
have difficulty using them. The prototypic pants created for the present study required the user to pull on a string attached to each side of the back of the underpants. Stroke patients are often paralyzed in both the upper and the lower extremities on one side; not only may they be unable to pull the string on the affected side with the hand on that side. But they may also be unable to reach that string with the unaffected hand. Therefore, it is still likely to be difficult for stroke patients to use the improved open-crotch underpants. The second problem was that patients with toileting problems are reported to be markedly psychologically affected by receiving toileting care ${ }^{1)}$. Many stroke patients with toileting problems have unstable or poor standing balance. Developing underpants that can be used by stroke patients could reduce the psychological burdens of toileting care and help slow the progress of dementia at the same time. These toileting aids would help promote independence when toileting and improve quality of life ${ }^{11)}$. However, in this study we could not examine the return to the original state. Therefore, we intend to further improve our open-crotch underpants so that stroke patients can use the hand on their unaffected side to open both sides of the crotch. In a future study, we also intend to determine the effects and efficacy of the underpants, including the return to the original state, in stroke patients.

\section{ACKNOWLEDGEMENT}

We sincerely thank all the participants for their cooperation in this study.

\section{REFERENCES}

1) Yu LC, Kaltreider DL, Hu T, et al.: The ISQ-P tool: measuring stress associated with incontinence. J Gerontol Nurs, 1989, 15: 9-15. [Medline] [CrossRef]

2) Sakurai H, Tsujimura $T$, Sugiura $Y$, et al.: Determinants of return to home after stroke: an analysis based on FIM Scores. J Phys Ther Sci, 2011, 23: 283-287. [CrossRef]

3) Okuno Y, Miyasaka T, Dobashi K: Factors influencing the outcome of acute rehabilitation: functional independence measure assessment at discharge. J Phys Ther Sci, 2012, 24: 491-494. [CrossRef]

4) American Occupational Therapy Association: Occupational therapy practice framework: Domain \& process (3rd ed). Am J Occup Ther, 2014, 68: S1-S48.

5) Keith RA, Granger CV, Hamilton BB, et al.: The Functional Independence Measure; A New Tool for Rehabilitation. Advances in clinical rehabilitation2. New York: Springer, 1987, pp 6-18.

6) Walker MF, Lincoln NB: Reacquisition of dressing skills after stroke. Int Disabil Stud, 1990, 12: 41-43. [Medline] [CrossRef]

7) Walker MF, Lincoln NB: Factors influencing dressing performance after stroke. J Neurol Neurosurg Psychiatry, 1991, 54: 699-701. [Medline] [CrossRef]

8) Gillen G, Burkhardt A: Stroke Rehabilitation: A Function-Based Approach. St. Louis: Mosby, 1998, pp 69-89.

9) Koike Y, Koeda S, Sumigawa K, et al.: Need for, and possibility of, training in seated dressing during toileting in stroke patients with poor standing balance. Igaku To Seibutsugaku, 2013, 157: 688-695.

10) Tacao FC: LTD. http://www.tacaof.co.jp/products/products.php?id=375 (Accessed Oct. 3, 2014) (in Japanese).

11) Parker MG, Thorslund $M$ : The use of technical aids among communitybased elderly. Am J Occup Ther, 1991, 45: 712-718. [Medline] [CrossRef] 